\title{
Numerical simulation of shock trains in a 3D channel
}

\author{
Romain Fiévet*, Heeseok $\mathrm{Koo}^{\dagger}$ and Venkat Raman ${ }^{\ddagger}$ \\ Department of Aerospace Engineering, The University of Michigan, Ann Arbor, MI 48109-2102, USA
}

\author{
Aaron H. Auslender \\ NASA Langley Research Center, Hampton, VA United States
}

\begin{abstract}
A dataset of normal shock trains in a rectangular cross-section channel has been created from Direct Numerical Simulations (DNS) in an effort to quantify the impact of inflow confinement ratio on the shock train structure. To this end, only the inlet boundary layer momentum thickness was varied while the bulk inflow and outflow conditions remained constant. The fully-resolved 3D turbulent boundary layer inflows correspond to atmospheric air isentropically expanded to Mach 2 and were obtained from auxiliary DNS. The simulations show that a change of inflow confinement ratio has a nonlinear impact on the shock train location, with a reduction in boundary layer momentum thickness leading to a displacement of the shock train downstream inside the isolator. As expected, an increase in boundary layer momentum thickness results in a reduction of the normal-like portion of the lambda-shock structures in the tunnel core. This leads to more numerous but weaker bifurcating shocks as well as an increase of the shock train length. It is also found that the growth rate of the boundary layer past the first bifurcating shock is dependent on both the inflow momentum thickness and the relative speed of the shock train compared to the bulk flow. When the inflow boundary layer thickness is varied temporally, the complex shock train response depends strongly on the excitation frequency. Its location along the tunnel is as expected more sensitive to lower frequencies while the shock train length exhibits a band-pass filter behavior.
\end{abstract}

\section{Introduction}

In dual-mode scramjet engines, the pre-combustion isolator section contains a shock train characterized by a core supersonic flow and a subsonic boundary layer dominated flow. Shock trains provide compression through not only a series of shock structures but also through flow confinement due to the growth of the near-wall subsonic region. The pressure and temperature gain through this shock train is critical for ensuring efficient combustion further downstream in the flow path. Hence, understanding the stability of shocks in confined supersonic flows is of interest for dual-mode operation.

In constant area channels, a supersonic flow with a turbulent boundary layer exhibits a range of flowstructures based on the inlet Mach number $M a$. At low supersonic speeds $(M a<1.2)$, a normal shock transitions the flow to a subsonic state. This normal shock becomes increasingly curved with increase in flow speed, and at velocities greater than $M a=1.5$, the single normal shock bifurcates and forms a series of shocks, referred to as a shock train. More importantly, the turbulent boundary layer undergoes separation leading to an axial reduction in the size of the core supersonic flow. This flow restriction, or confinement, through the formation of a subsonic region is critical in determining the structure of the shock train. The confinement effect on shock trains is directly related to the incoming boundary layer properties. For instance, Carroll and Dutton ${ }^{1}$ have shown that as the ratio of inflow boundary layer thickness to duct height increases,

\footnotetext{
${ }^{*}$ PhD Student, AIAA Student Member.

${ }^{\dagger}$ Post-Doctoral Research Fellow, AIAA Member.

¥Associate Professor, AIAA Senior Member,

Copyright (C) 2016 by Romain Fiévet. Published by the American Institute of Aeronautics and Astronautics, Inc. with permission.
} 
both the number of shocks and the spacing between the shocks increases. At the same time, the bifurcation associated with the leading shock reaches more towards the centerline as confinement ratio, defined as the ratio between the boundary layer and channel heights increases. Further, the effect of the confinement ratio decreases as the Mach number increases.

Such supersonic isolators have been studied using both computational and experimental resources. ${ }^{2-7}$ The experimental studies have mainly used pressure measurements, laser Doppler velocimetry, and Schlieren images to reconstruct the shock-train structure. ${ }^{1,3,8,9}$ Waltrup and Billig ${ }^{3}$ analyzed pressure traces to propose an empirical relation between shock train length and wall pressure. In this work, the shock train

length was found to have a $R e_{\theta}^{1 / 4}$ dependence, where $R e_{\theta}$ is the momentum-thickness based Reynolds number at the inlet of the isolator section.

The computational studies of isolators are typically of two kinds. The first utilizes an inlet ramp to create an initial incident shock that is relatively fixed in time. This yields a steady attached shock train, which is fixed to the foot of the first reflected shock and can be expected to be adequately described by inviscid theory. Of course, further downstream, the reflected shocks are weaker and are affected by the turbulent flow structure inside the isolator. Koo and $\operatorname{Raman}^{4}$ used a large eddy simulation (LES) to simulate a Mach 5 supersonic inlet-isolator with unstart, which was caused by an increase in back pressure at the outlet. Overall, they concluded that the LES approach, with relatively simple wall models, is able to capture the overall shock structure for both static and unstart cases. However, there was a significant difference in the timescales associated with unstart, where the LES predicted a faster shock propagation speed compared to the experiments.

The second set of isolator simulations involve a normal shock-train, ${ }^{5,6}$ which is more relevant to the dualmode scramjet regime. Here, the initial shock bifurcation is caused by flow confinement and the boundary layer. Morgan et al. ${ }^{6}$ have used LES to compute such a normal shock-train in a three-dimensional rectangular channel, corresponding to the experimental setup of Carroll and Dutton. ${ }^{9}$ Even at an order of magnitude lower Reynolds number than experiments, the shock structure was found to be very similar to the experiment. Further, the presence of the side wall as opposed to a spanwise periodic boundary was found to affect the evolution of lower-wall pressure profiles. In particular, the pressure reached a maximum and then slowly decayed towards the exit, indicating a loss of compression beyond the peak point. Overall, such studies have shown that LES-type resolution for reduced Reynolds numbers (as compared to practical applications) still reproduce most of the critical features of a shock-train.

With this background, the focus of this work is in understanding the effect of the inflow boundary layer on shock train formation. As opposed to prior studies, the back pressure at the exit of the isolator is fixed in these simulations, but the incoming flow characteristics are altered to reflect flight path effects. A high-resolution numerical approach is followed, where the smallest near-wall features are resolved. Finally, unsteady effects caused by temporal variations in inflow boundary layer are studied to understand the response of the isolator to perturbations in inflow. The rest of the sections are organized as follows: Sec. II discusses the flow configuration and numerical details, Sec. III presents the results on the effect of boundary layer thickness on shock train evolution. Sec. IV is dedicated to temporal effects on shock train motion, as the inflow boundary layer momentum thickness oscillates at different frequencies.

\section{Flow configuration and numerical details}

The simulation configuration is chosen to replicate the University of Michigan expansion tunnel facility, where a similar study on how back pressure variation affects the shock train behavior is being conducted. ${ }^{10,11}$ The simulation domain consists in a $69.8 \times 57.2 \mathrm{~mm}$ constant-area rectangular channel to which a back pressure is applied at the outlet as a numerical boundary condition. The back pressure is fixed to $48800 \mathrm{~Pa}$ corresponding to $75 \%$ of a normal shock pressure rise for this Mach number. To be consistent with other studies, the channel half height $h=34.9 \mathrm{~mm}$ is used as a reference length throughout the paper. The inflow is at Mach 2, and is injected as a fully developed turbulent channel boundary layer. The computational domain spans $856 \mathrm{~mm}$ in the streamwise direction (roughly $25 \mathrm{~h}$ ), which is sufficient to fully accommodate the shock train for the range of conditions studied here.

As previously discussed, the focus of this study is to understand the impact of inflow boundary layer conditions on shock train formation and response. For this purpose, three different inflow boundary layers generated from an auxiliary channel flow simulation are used. These inflows were first generated on a very fine grid with the same rectangular cross-section but a shorter length, with streamwise periodic boundaries 


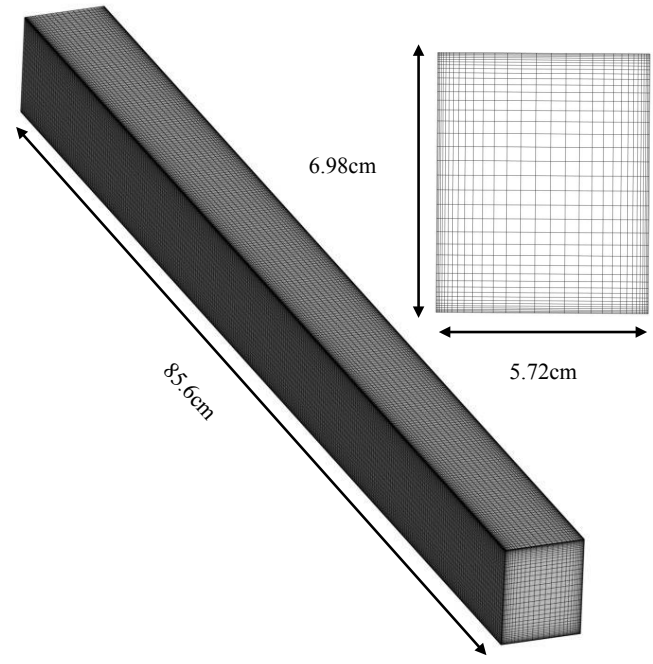

Figure 1. Computational domain and grid, with $1 / 4^{t h}$ nodes in every direction plotted.

and a DNS-like resolution. Time-correlated planes of inflow data were created by sampling this DNS when the channel had boundary layers with relevant momentum thickness. Since the momentum thickness grows in a periodic channel to occupy the entire cross-section, such sampling can be carried out only over finite time, where the growth rate within the sampling window was neglected. In general, these inflow samples spanned 1/4 residence time in the actual isolator, where this time was computed based on the length of the isolator and the core velocity. The inflow conditions and the corresponding parameters are shown in Tab 1. The resolution was carefully chosen such that no extraneous compression shocks are generated at the inlet due to a mismatch between the isolator and channel computational grids. Figure 2 shows an instantaneous snapshot of the Mach number at the inflow and the near wall mean flow structure for different spanwise and wall-normal locations. It is seen that near the middle of each side, the profiles are similar to fully developed boundary layer flow. Closer to the corners, the interaction of the boundary layers will cause the profiles to differ from the conventional log-law profiles. The inertial sublayer is correctly resolved in both cases.

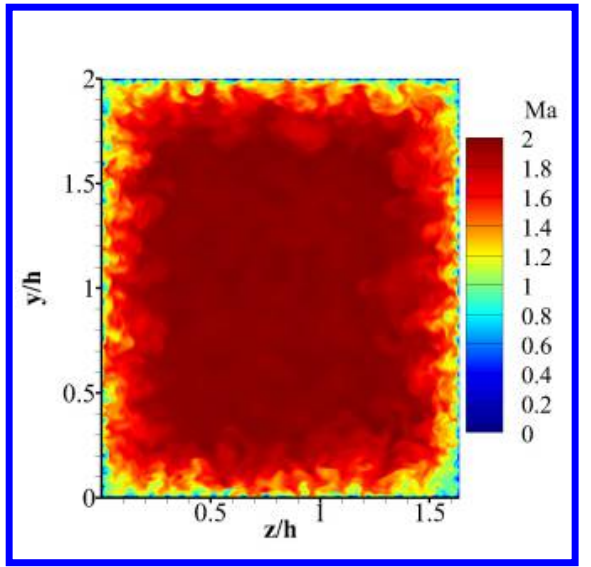

(a)

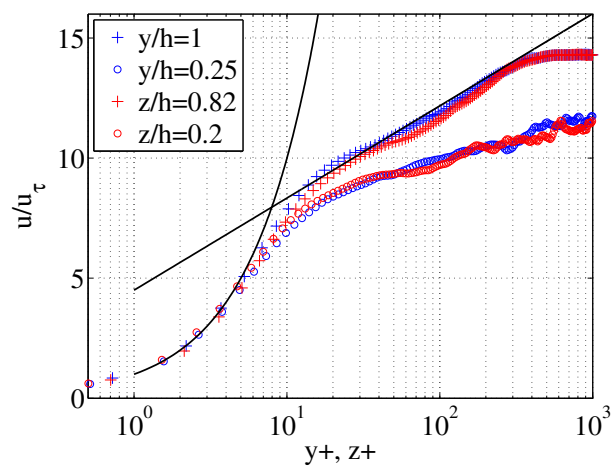

(b)

Figure 2. Case B inflow 3D turbulent boundary layer. (Left) Mach number snapshot and (right) wall-normal velocity profiles from side walls (blue) and bottom walls (red) at $(+)$ half channel and $(\circ)$ eighth channel heights.

The computational domain is discretized using an orthogonal grid system spanning $\left(n_{x}, n_{y}, n_{z}\right)=(2048,320,256)$ points. The grid points are clustered near the walls such that the spatial resolution based on wall units is $\{\Delta x+=18, \Delta y+=0.9-18, \Delta z+=0.9-18\}$, where the variations in the wall-normal and spanwise directions indicates the range for the three different simulations. The wall viscous length scale was computed to be $23 \mu \mathrm{m}$ based on the inflow boundary layers. 
Table 1. Flow conditions for the three different inflow considered

\begin{tabular}{lccccccc} 
Case & $P[k P a]$ & $T[K]$ & $M a$ & $\delta[\mathrm{mm}]$ & $\delta / h$ & $\theta[\mathrm{mm}]$ & $R e_{\theta}$ \\
\hline$A$ & 14.1 & 170.0 & 1.96 & 8.8 & 0.31 & 1.3 & 4530 \\
$B$ & 14.5 & 171.0 & 1.96 & 9.8 & 0.34 & 1.8 & 6380 \\
$C$ & 14.8 & 172.0 & 1.97 & 11.9 & 0.42 & 2.2 & 7900
\end{tabular}

The simulations were performed using the in-house compressible flow solver, UTComp, which has been extensively validated. ${ }^{12-15}$ The solver uses a fifth-order WENO scheme ${ }^{16}$ with characteristics reconstruction to compute the nonlinear momentum terms. A fourth-order central scheme is used for the viscous and diffusion terms, and the walls are treated adiabatically. The viscosity is determined using Sutherland's law and is multiplied by 4 to obtain a more tractable Reynolds number. The thermal diffusivity is obtained from a constant Prandtl number (0.7). Further details on the flow solver are provided in. ${ }^{4,17}$ The simulations were initialized from a quiescent flow and continued until all transients were removed from the computational domain. Statistics were sampled over 2.5 flow-through timescales $\tau_{c}\left(1 \tau_{c}=2.1 \mathrm{~ms}\right)$, running on 4800 cores for 120 hours.

\section{Effect of inflow boundary layer thickness}

The three cases (A, B, and C, see table. 1) were simulated using the procedure described in Sec. II. Figures 3 and 4 show the instantaneous Mach number and density gradient magnitude contours for case B. Similar to experimental predictions, a series of shock structures is present. Once the primary shock is formed, the boundary layer thickens, leading to an increasing volume of subsonic flow with axial distance. Past the terminating (last) shock, a mixing region is observed, where both subsonic and supersonic flow are present. The simulation shows strong oscillations in the locations of the leading shock, in agreement to the observations corresponding to conventional shock-boundary layer interactions. ${ }^{18,19}$ Overall, the simulation reproduces the essential features of a shock train. Figure 4 also highlights the evolution of the leading shock as a function of confinement ratio. Case A's leading lambda-shock presents a larger normal-like portion than that in case $\mathrm{C}$, resulting in a larger subsonic region behind it as seen in figure 5 .

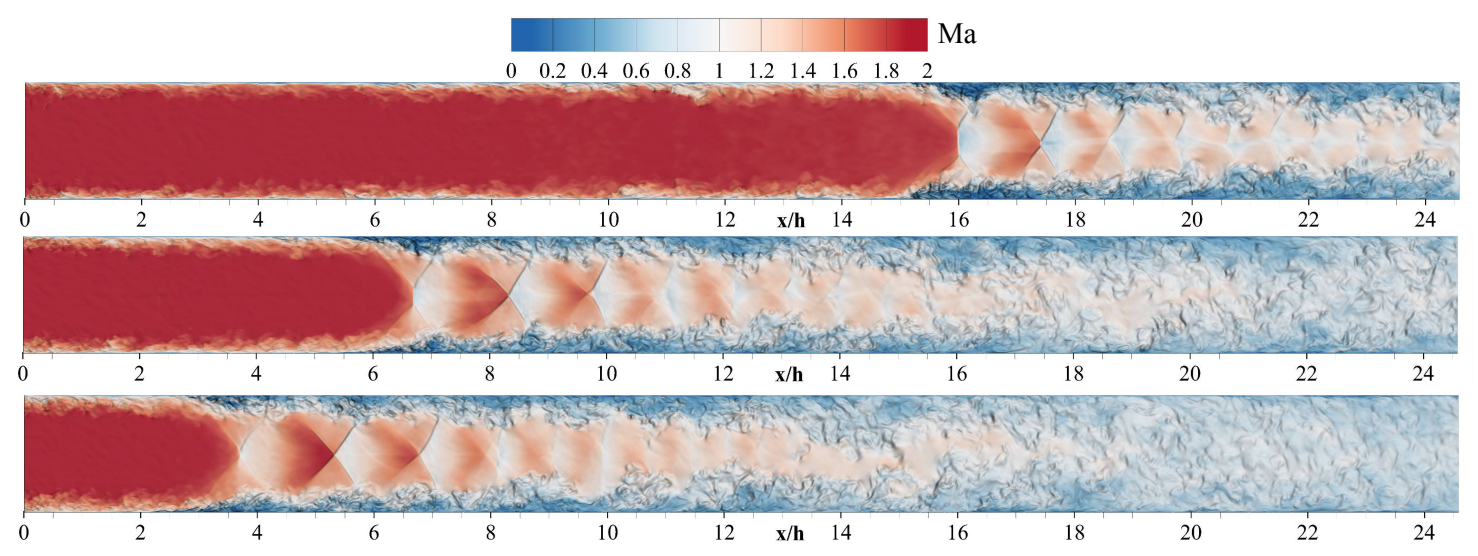

Figure 3. Instantaneous snapshots of Mach number for (top) case A, (center) case B and (bottom) case C. 


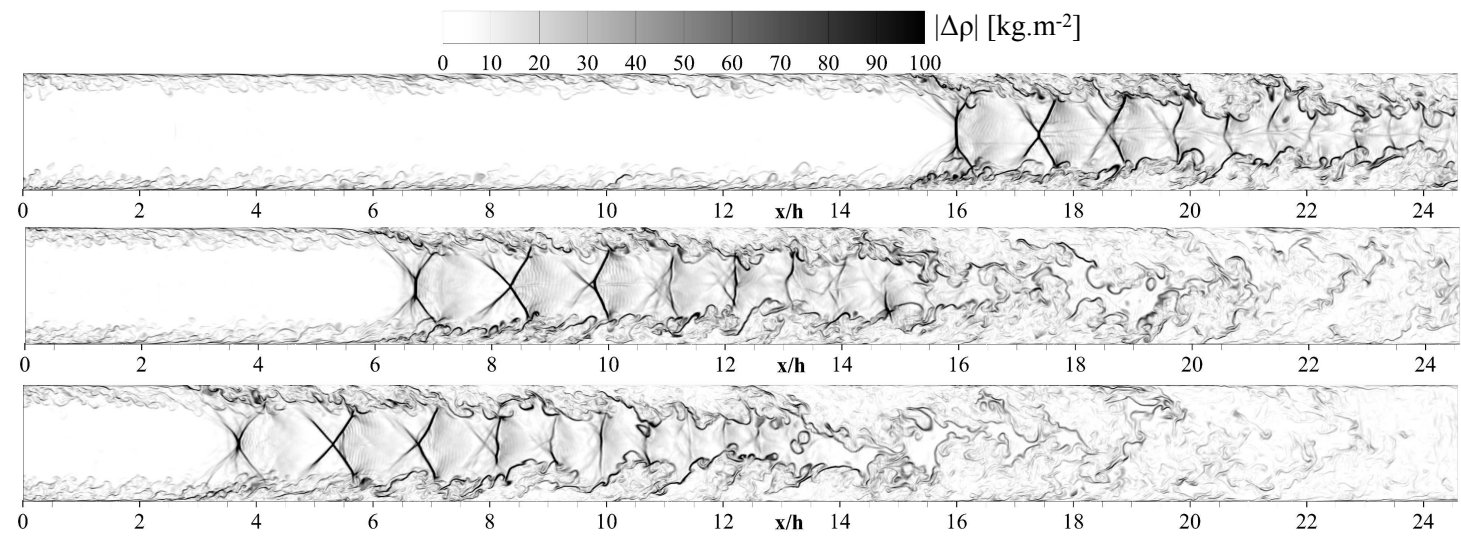

Figure 4. Instantaneous snapshots of density gradient magnitude for (top) case A, (center) case B and (bottom) case C.
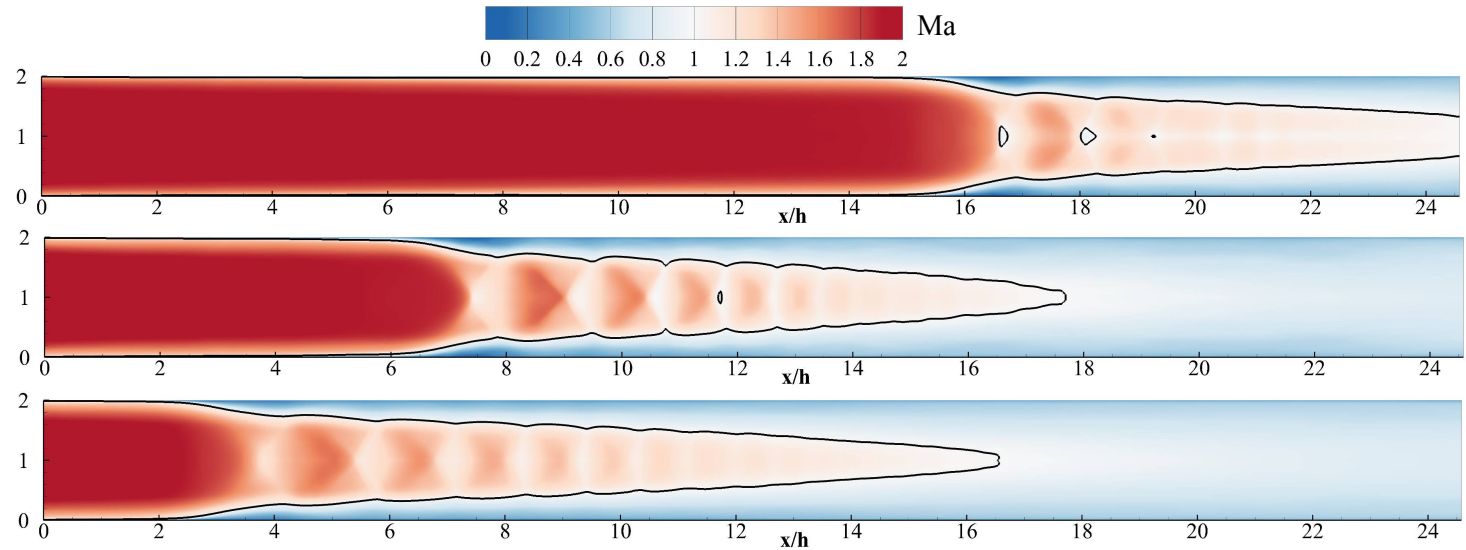

Figure 5. Averaged contours of Mach number with sonic line in black for (top) case A, (center) case B and (bottom) case C.

Figures 5 and 6 show the time-averaged contours Mach number and pressure. The shocks are weaker as compared to the instantaneous values, consistent with the averaging over spatially oscillatory structures. This is further evidenced in the Mach number contours that show regularly spaced supersonic flow region with near sonic or subsonic flow regions in between. It should be noted that the Mach number contours are evaluated based on the average properties of the fluid, and is not equal to the average Mach number. Hence, even if the flow is supersonic instantaneously everywhere in the core, it might appear subsonic due to the spatial oscillations of the shock structures as well as this averaging procedure (we note that in an "averaged" simulation approach, it is this Mach number that will be reproduced even though the underlying flow exhibits a vastly different structure). Although the pressure gradient shows strong fluctuations near the centerline, it progressively increases to the applied back pressure value. This would indicate that the isolator is longer than needed, where frictional losses lead to slight loss of compression.

As expected, the shock train moves downstream with a reduction in inflow boundary layer thickness. The initial shock location is also highly sensitive to the boundary layer thickness, with a $100 \%$ change in axial location for a $25 \%$ change in $\delta / h$. As the boundary layer thickness increases, the shock structures are found to be weaker, so the number of shocks increases. On the other hand, a reduction in boundary layer thickness pushes the initial shock structure further downstream and compresses the shock train spatially. The boundary layer growth-rate past the first shock structure is also dependent on the boundary layer thickness. For larger thicknesses, the growth rate is lower since the isolator has sufficient axial length to reach the prescribed back pressure. In this sense, the volume of subsonic region controls the rate of increase of pressure. Note that the density gradients across the shock structure are roughly equal for all cases, indicating that the growth of the subsonic region plays a bigger role in affecting the axial compression rate.

Similar observations can be made from figure 6, which shows the growth of the time-averaged pressure profiles along the wall and the centerline. The centerline profiles show the successive compression and 

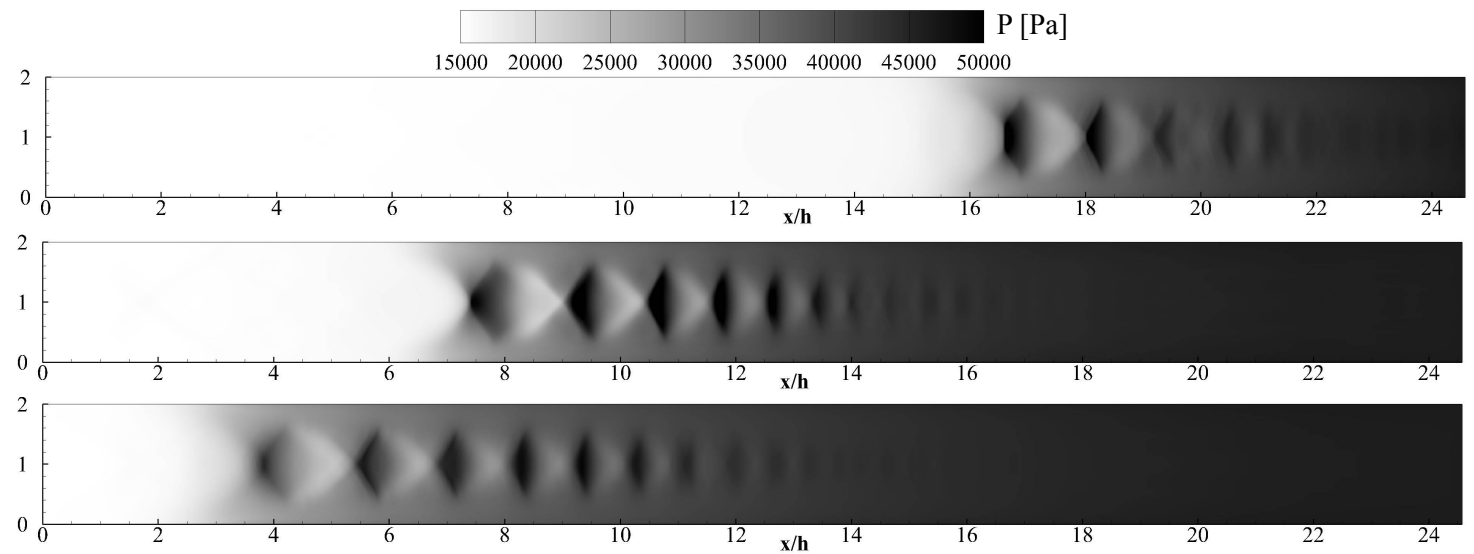

Figure 6. Averaged contours of static pressure for (top) case A, (center) case B and (bottom) case C.

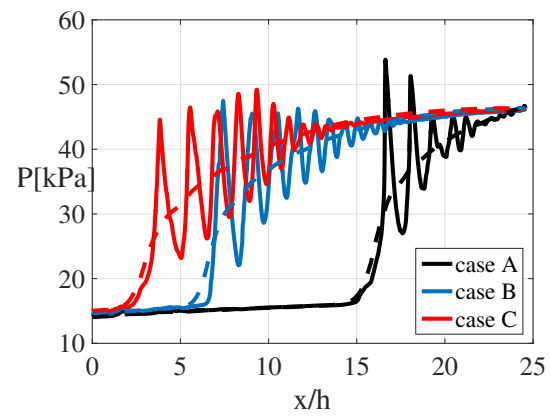

Figure 7. Centerline (solid) and wall (dashed) static pressure profiles for all cases.

expansion associated with the shock structures, while turbulent diffusion in the boundary layers remove these pressure fluctuations near the walls. Past the last shock structure, both the core and boundary layer pressure profiles match, which marks the mixing region of the isolator. The pressure profiles for the three cases show the nonlinear effect of boundary layer thickness on the initial shock location. The change from case $\mathrm{B}$ to $\mathrm{C}$ fails to generate a large change in initial position, while case A shows a shock structure that has moved much farther downstream. This is in contrast to the nearly square root dependance implied by the empirical correlation of Waltrup and Billig. ${ }^{3}$ As explained earlier, the main reason for this departure is the growth rate of the boundary layer, which is also dependent on the distance between the initial shock location and the exit of the isolator. Another striking feature is the strength of the first shocks for case A which is high enough to make the flow subsonic around the centerline as can be seen in figure 5 by looking at the sonic line contours. Again, this is consistent with the fact that the shock train has to accommodate the same back pressure condition over a shorter length.

All shock trains are also observed to be highly unsteady and shift within the isolator at a very low frequency. This characteristic unsteadiness is a known feature of shock-boundary layer interactions, ${ }^{18}$ and is shown in figure 8 where 16 frames are equally spaced in time to cover $2 \tau_{c}$ for case A. The contours of numerical Schlieren highlight both the shape of the shocks and the turbulent boundary layer, giving insight into their coupling mechanics. Indeed, the magnitude of the boundary layer separation depends on the pressure gradient between the wall and the centerline, and is therefore a good indicator of the first shock strength. As can be seen from figure 8, the shock train first moves downstream towards the outlet, and comes back upstream against the flow. While the shock train moves with the flow its relative speed decreases, weakening the shocks. On the other hand, when the shock train moves upstream it increases its relative Mach number and shock strength. A crude evaluation of the apparent Mach number evolution can be made assuming from figure 8 a sinusoidal oscillation of amplitude of 1 channel height and of frequency $f=100 \mathrm{~Hz}$. This leads to a relative convective speed of maximum amplitude $2 \pi h f$, resulting in a range of apparent Mach number $M a_{a p p} \in[1.83 ; 2.17]$, oscillating around the bulk Mach number $M a=2$. This broad range of apparent Mach number changes the nature of the shock train as described in Matsuo $a l^{8}{ }^{\text {from a }}$ normal shock train to an oblique shock train type (smaller normal part in the leading shock, thicker boundary 

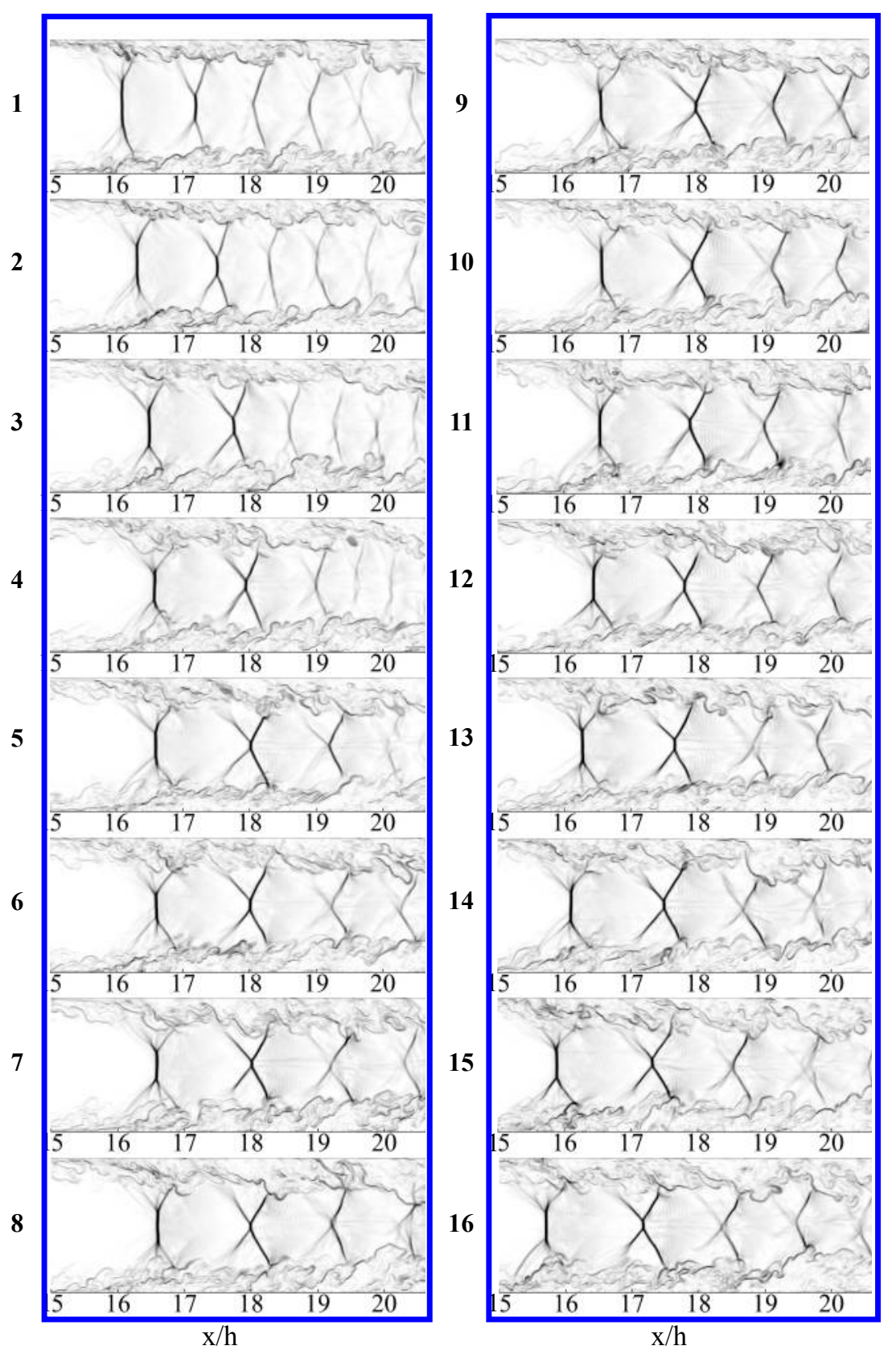

Figure 8. Snapshots of density gradient of the shock train foot for case A ordered from 1 to 16 sampled over a duration of $2 \tau_{c}$.

layer and larger distance between shocks). In figure 8 frames 1-4 correspond to a weak strand, 5-8 to a steady strand, and 9-16 to a strong one. A comparison between frames 1 and 16 validates the previous statements as both the distance between shocks and the turbulent boundary layer thickness increase, which is characteristic of a higher Mach number shock train. Due to the large timescales involved with this phenomena, statistics might differ wether they are sampled in this oblique/normal or strong/weak shock train half-period.

\section{Effect of inflow boundary layer thickness fluctuations on shock trains}

A practical consequence of this high nonlinearity of shock location with boundary layer thickness is the effect of any inflow disturbances on shock train behavior. In practical designs, the downstream combustor 
section will respond to shock train movement, and the fixed back pressure condition may not be feasible. However, if this pressure is indeed fixed, the response of the isolator provides a sense of the dynamics of the shock train. In this regard, Klomparens al. ${ }^{11}$ have varied isolator back pressure to study the response of the isolator. The frequency of such variations in the experiments (roughly 0.59-3.57 Hz) is too low for high fidelity numerical calculations. Here, a set of high frequency cases are explored to understand the key processes that control the shock train response. Due to the low-frequency components we seek to capture these new simulations have to cover a time of approximately hundred $\tau_{c}$. Therefore it is necessary to reduce the computational expense by reducing the grid resolution. The coarse grid resolution halved the point in every direction corresponding to a resolution of $\{\Delta x+=36, \Delta y+=1.8-36, \Delta z+=1.8-36\}$. With the boundary condition corresponding to case $\mathrm{B}$, the shock train was found relatively steady when the coarse grid was used. Figure 9(a) shows the centerline pressure space-time diagram that spans over $25 \tau_{c}$ in time. The unsteadiness observed in the previous section disappears and conveniently creates a steady shocktrain which serves as a baseline. Indeed, as no oscillations are found in the shock train length and position we can infer that any unsteadiness observed in the rest of the analysis is the exclusive shock train response to the inflow oscillations. Grid convergence was verified in figure $9(\mathrm{~b})$ which testifies the ability of the coarse grid to capture the shock train structure, length and positions, although it underestimates the first shock strength. For the rest of the analysis, this shock strength is not directly relevant.

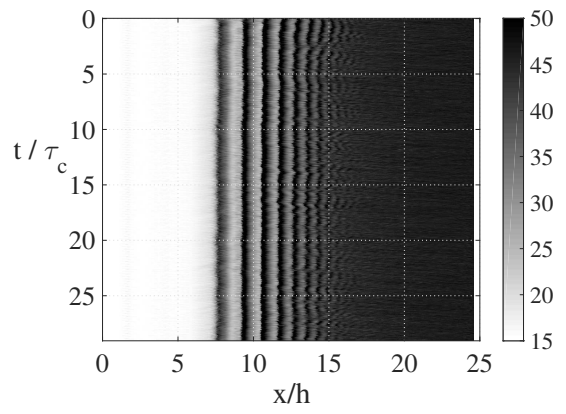

(a)

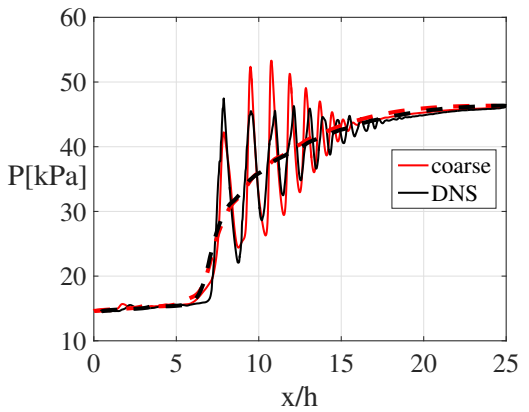

(b)

Figure 9. (a) Space-time diagram of case B on the coarse grid (b) Grid convergence between the DNS and coarse grid.

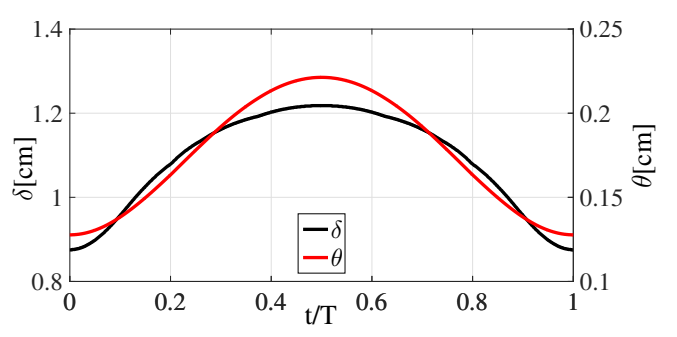

Figure 10. Inflow boundary layer oscillation over one period.

The inflow variation is introduced by temporally interpolating between the inflow fields for case A and C. Figure 10 shows the variation in momentum and boundary layer thicknesses over one period. Five different cases, corresponding to cycling frequencies of 20,100, 200, 500 and $1000 \mathrm{~Hz}$ are simulated. In each case, the wall pressure profile is used to estimate the shock train length. For instance, Figure 11(a) shows the instantaneous pressure trace as well as a filtered trace. The foot of the shock is estimated based on the earliest location where the filtered pressure rises to $10 \%$ of the difference between the inflow and back pressure. The mid-point of the shock-train is determined as $50 \%$ of that same value, while its tail is located at $80 \%$. The shock train length is the distance between $10 \%$ and $80 \%$ pressure rise locations. Additionally, the location of the first 4 shocks is used to quantify the shock train response to these oscillations, using a shock-detecting algorithm as demonstrated in figure 11(b).

$$
\chi_{s t}(t)=\frac{\left(x_{80}-x_{10}\right)(t)}{\left(x_{80}-x_{10}\right)(t=0)}
$$




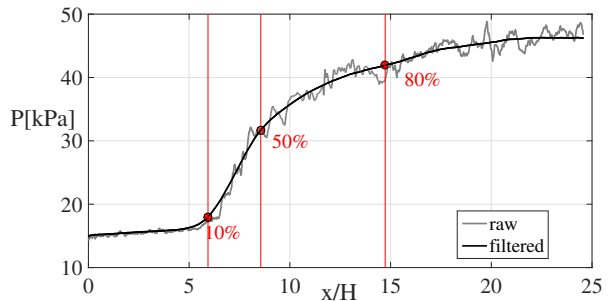

(a)

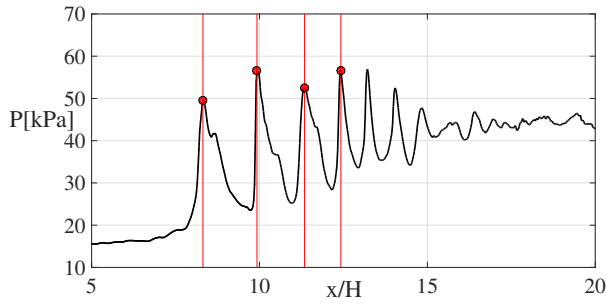

(b)

Figure 11. (a) Filtering process for the wall pressure signal and identification of 10,50 and $80 \%$ back pressure rise locations. (b) Typical raw centerline pressure profile and location of the first 4 shocks.
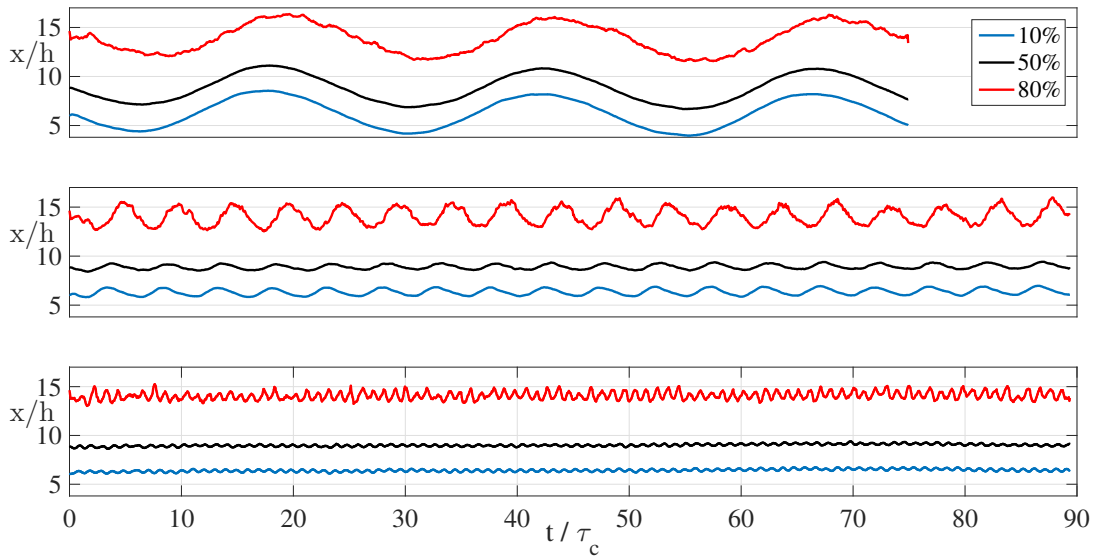

Figure 12. Time-varying locations of the wall pressure 10, 50 and $80 \%$ back pressure rise for excitation frequencies of (top to bottom) $20 \mathrm{~Hz}, 100 \mathrm{~Hz}$ and $500 \mathrm{~Hz}$.

$$
\xi_{s t}=100 \max \left(\frac{\chi_{s t}(t)-1}{2}\right)
$$

First, the temporal evolution of the 10,50 and $80 \%$ back pressure rise locations is analyzed. Figure 12 presents these signals for the 20,100 and $500 \mathrm{~Hz}$ cases. The $20 \mathrm{~Hz}$ case presents similar amplitude oscillations for all 10, 50 and $80 \%$ signals, although the shock train foot and tail are slightly out of phase due to the small reaction lag for the instability to propagate downstream. An essentially frozen state of the $500 \mathrm{~Hz}$ case indicates that $500 \mathrm{~Hz}$ appears to be a too high frequency to be accommodated in the system. However, the $100 \mathrm{~Hz}$ intermediary case presents some interesting nonlinearity as it is not merely a blend of the lowfrequency and high-frequency solutions. The shock train foot amplitude oscillation does decrease compared to the $20 \mathrm{~Hz}$ case, but the tail response stays as strong and is out of phase. $\chi_{s t}(t)$ defined in equation (1) is plotted in figure 13 for these 3 frequencies. Alongside, the time signal is plotted $\xi_{s t}$ defined in equation (2) as the maximum oscillation amplitude of the shock train for a particular excitation frequency. This highlights the string-like behavior of the shock train to upstream instabilities as the frequency-response resembles a band-pass filter of characteristic frequency $\left.\left.f_{c} \in\right] 20 \mathrm{~Hz} ; 100 \mathrm{~Hz}\right]$.

$$
\begin{gathered}
\tilde{S}_{s, i}(f)=\frac{S_{s, i}(f)}{S_{s, i}\left(f=f_{i}\right)} \\
\tilde{A}_{s, i}=\frac{S_{s, i}\left(f=f_{i}\right)}{h}
\end{gathered}
$$

We can now focus our attention on the locations of the first 4 shocks, extract their time signal and perform a spectral analysis for all cases. The time-signals are presented in figure 14 to illustrate the shock train response. Let $\tilde{S}_{s, i}(f)$ be defined in equation (3) as the single-sided amplitude spectra of the shock locations for excitation frequency $f_{i}$ normalized by its value at $f_{i}$. Results of the spectrum analysis are presented in figure 15. It is noted that the most energetic frequencies match the excitation frequencies 

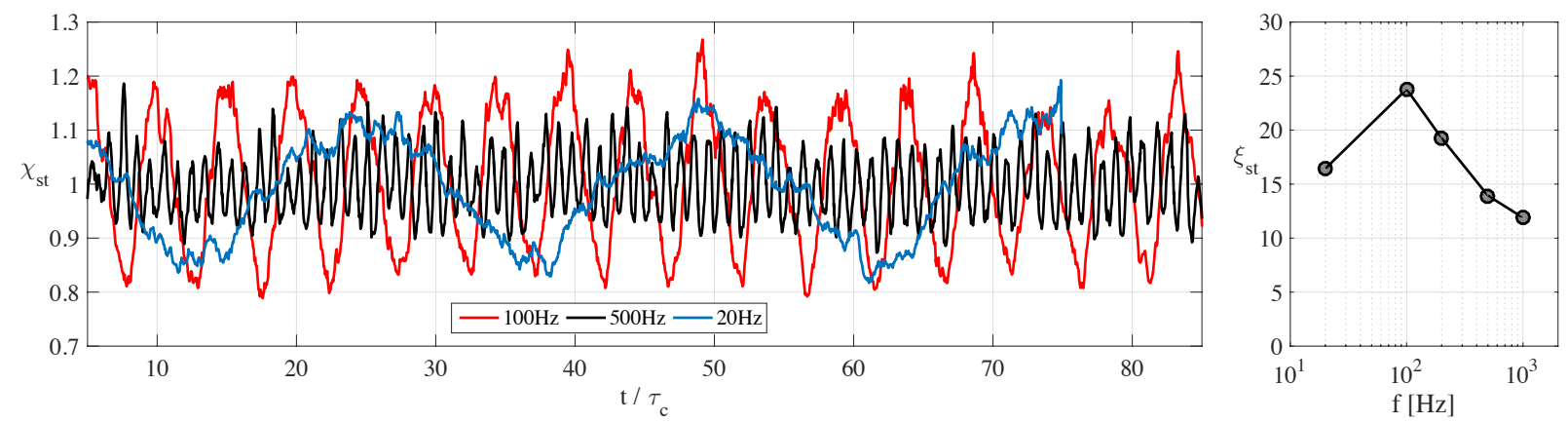

Figure 13. (left) shock train length as a function of time for 20,100 and $500 \mathrm{~Hz}$ excitation and (right) normalized amplitude oscillation of the shock train length for all excitation frequencies.
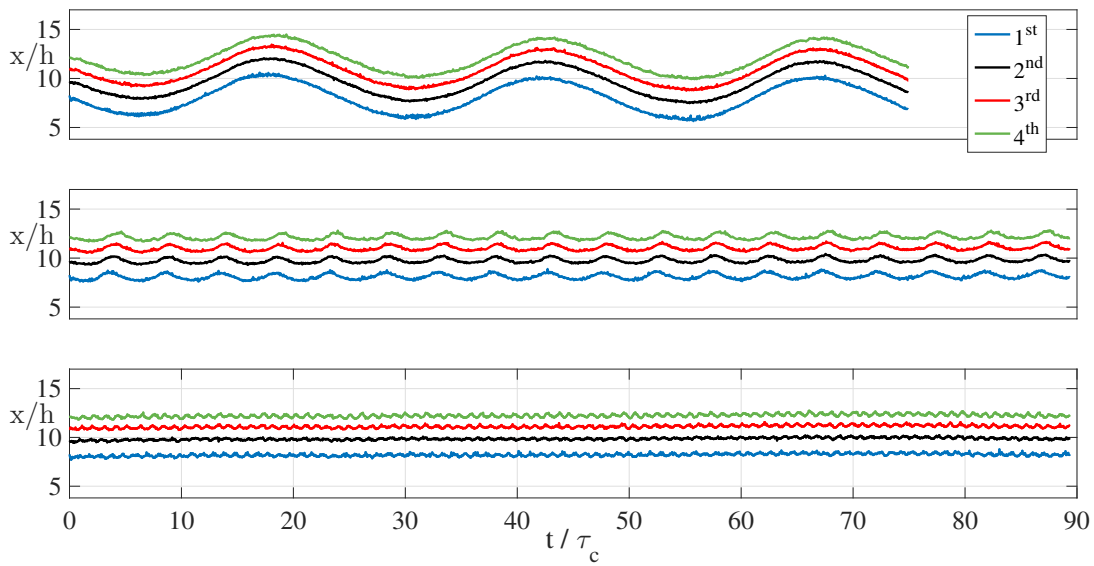

Figure 14. Time-varying locations of the first 4 shocks along the centerline for excitation frequencies of (top to bottom) $20 \mathrm{~Hz}, 100 \mathrm{~Hz}$ and $500 \mathrm{~Hz}$.

and their following harmonics. We can then define the amplitudes of shock oscillations for all excitation frequencies as $\tilde{A}_{s, i}$ in equation (4), which are plotted in figure 16. The plots show the expected low-pass shock train response to these upstream instabilities. As the frequency increases the shock train cannot accommodate the associated timescale and the shocks stay idle. However at the lowest frequency among those simulated the shock train response timescale becomes much smaller than the instability timescale and a smooth sinusoidal oscillation between case A and case C solutions is observed.

\section{Conclusions}

Direct numerical simulations of shock trains in a turbulent channel have been carried out. The database contains shock train data at different inflow conditions, characterized by variations in boundary layer thickness. The simulations show a nonlinear dependance of shock train position on boundary layer thickness, with larger confinements showing less sensitivity to confinement. Conversely, when the boundary layer thickness is small, the shock train is pushed towards the exit, leading to short but stronger compression regions. When the inflow conditions are varied over time, the shock train response is non-trivial and depends on the excitation frequency. With too high or too low excitation frequency, the shock train either stays nearly stationary or moves coincidently to the inflow variation. However when the frequency is $100 \mathrm{~Hz}$, the foot and the tail of the shock train fall out of phase and the shock train length becomes the maximum. This suggests that the shock train string-like behavior possesses a characteristic frequency which maximizes its unsteadiness, and potentially accelerate/cause unstart in a dual-mode scramjet engine. 


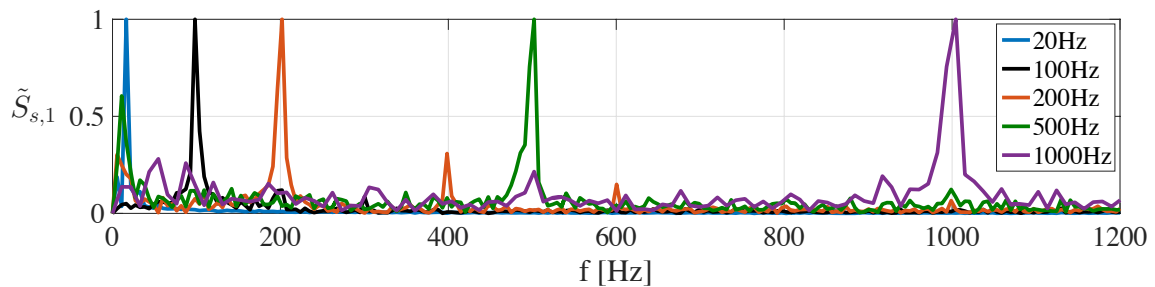

(a)

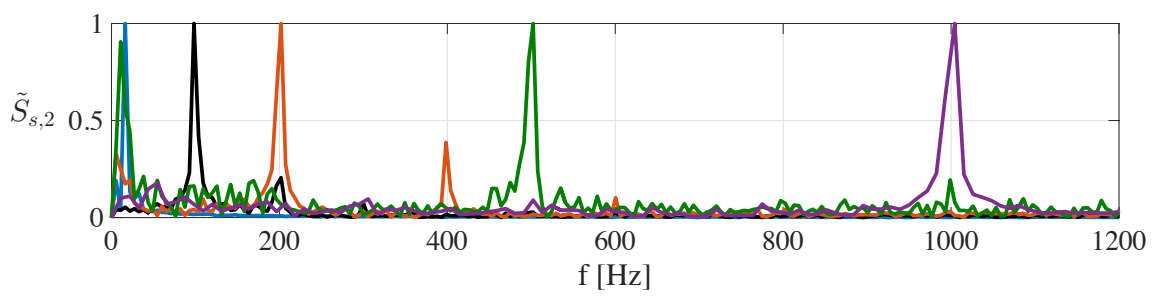

(b)

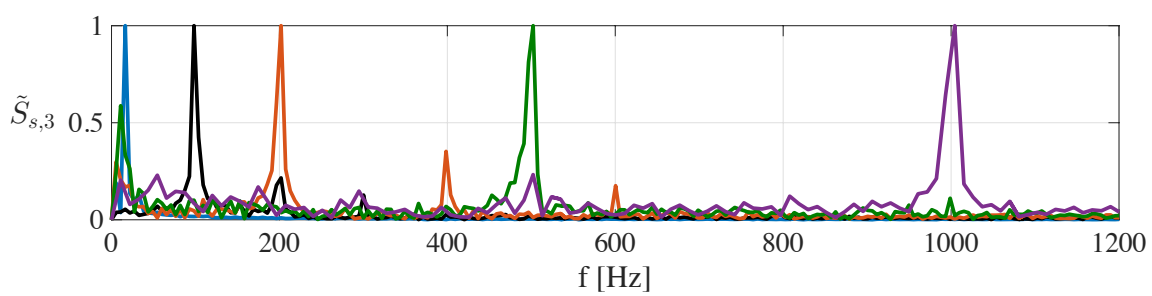

(c)

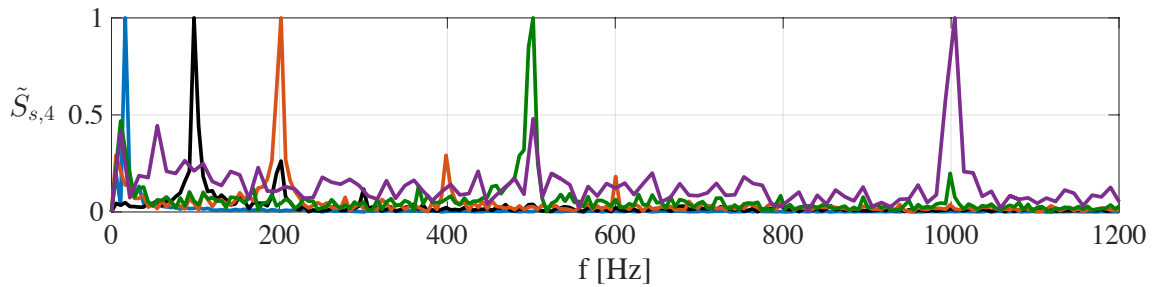

(d)

Figure 15. Normalized single-sided amplitude spectra of (a) first shock, (b) second shock, (c) third shock and (d) fourth shock location for all excitation frequencies.

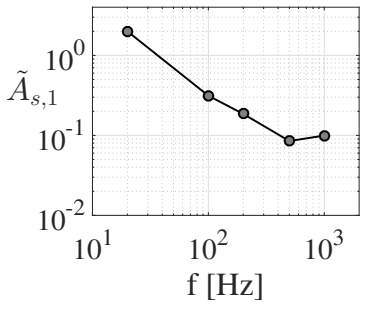

(a)

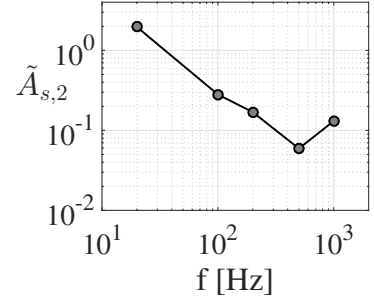

(b)

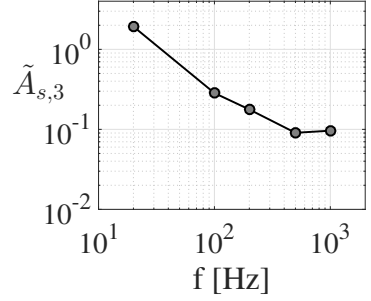

(c)

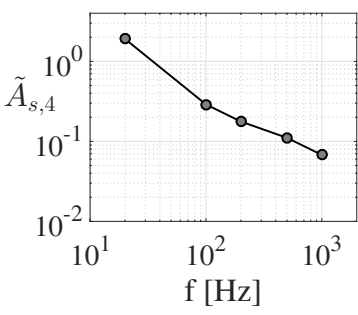

(d)

Figure 16. Oscillation amplitude of the (a) first shock, (b) second shock, (c) third shock and (d) fourth shock location depending on the frequency of excitation.

\section{Acknowledgements}

This work was financially supported through an AFRL grant FA9550-12-1-0460. The authors also gratefully acknowledge the generous allocation of computing time by NASA High-End Computing (HEC) Program 
through the NASA Advanced Supercomputing (NAS) Division at Ames Research Center.

\section{References}

${ }^{1}$ Carrol, B. and Dutton, J., "Characteristics of Multiple Shock-wave Turbulent Boundary-layer Interactions in Rectangular Ducts," Journal of Propulsion and Power, Vol. 6, No. 2, MAR-APR 1990, pp. 186-193.

${ }^{2}$ Sullins, G. and Mclafferty, G., "Experimental Results of Shock Trains in Rectangular Ducts," AIAA Paper 92-5104, 1992.

${ }^{3}$ Waltrup, P. J. and Billig, F. S., "Structure of Shock Waves in Cylindrical Ducts," AIAA Journal, Vol. 11, No. 10, 1973, pp. $1404-1408$.

${ }^{4}$ Koo, H. and Raman, V., "Large-Eddy Simulation of a Supersonic Inlet-Isolator," AIAA Journal, Vol. 50, No. 7, 2012, pp. $1596-1613$

${ }^{5}$ P. A. T. Cocks, C. Bruno, J. M. D. and Haas, M., "IDDES of a Dual-Mode Ethylene Fueled Cavity Flameholder with an Isolator Shock Train," 51st AIAA Aerospace Sciences Meeting, No. AIAA-2013-0116, 2013.

${ }^{6}$ B. Morgan, K. D. and Lele, S. K., "Large-Eddy Simulations of a Normal Shock Train in a Constant-Area Isolator," AIAA Journal, Vol. 52, No. 3, 2014, pp. 539-558.

${ }^{7}$ Carrol, B., Lopezfernandez, P., and Dutton, J., "Computations and Experiments for a Multiple Normal-shock Boundarylayer Interaction," Journal of Propulsion and Power, Vol. 9, No. 3, MAY-JUN 1993, pp. 405-411.

${ }^{8}$ Matsuo, K., Miyazato, Y., and Kim, H.-D., "Shock Train and Pseudo-Shock Phenomena in Internal Gas Flows," Progres in Aerospace Sciences, Vol. 35, 1999, pp. 33-100.

${ }^{9}$ Carrol, B. and Dutton, J., "Multiple Normal Shock-wave Turbulent Boundary-layer Interations," Journal of Propulsion and Power, Vol. 8, No. 2, MAR-APR 1992, pp. 441-448.

${ }^{10}$ Klomparens, R. L., Driscoll, J. F., and Gamba, M., "Unsteadiness characteristics and pressure distribution of an oblique shock train," 53rd AIAA Aerospace Sciences Meeting, No. AIAA-2015-0116, 2015.

${ }^{11}$ Klomparens, R. L., Driscoll, J. F., and Gamba, M., "Response of a shock train to downstream back pressure forcing," 54th AIAA Aerospace Sciences Meeting, No. AIAA-2016-0078, 2016.

$\checkmark{ }^{12}$ Koo, H., Donde, P., and Raman, V., "A Quadrature-based LES/Transported Probability Density Function Approach for Modeling Supersonic Combustion," Proceedings of the Combustion Institute, Vol. 33, 2011, pp. 2203-2210.

13Koo, H., Donde, P., and Raman, V., "LES-based Eulerian PDF approach for the simulation of scramjet combustors," Proceedings of the Combustion Institute, Vol. 34, No. 2, 2013, pp. 2093-2100.

$\checkmark{ }^{14}$ Donde, P., Koo, H., and Raman, V., "A multivariate quadrature based moment method for LES based modeling of supersonic combustion," Journal of Computational Physics, Vol. 231, No. 17, 2012, pp. 5805-5821.

${ }^{15}$ Fiévet, R., Koo, H., and Raman, V., "Numerical simulation of a scramjet isolator with thermodynamic nonequilibrium," 22nd AIAA Computational Fluid Dynamics Conference, 22-26th June 2015, Dallas, TX, USA, No. AIAA-2015-3418, 2015.

${ }^{16}$ Jiang, G.-S. and Peng, D., "Weighted ENO Schemes for Hamilton-Jacobi Equations," SIAM Journal of Scientific Computing, Vol. 21, No. 6, 2000, pp. 2126-2143.

${ }^{17} \mathrm{Koo}, \mathrm{H} .$, Large-eddy simulations of scramjet engines, Ph.D. thesis, The University of Texas at Austin, 2011.

$\checkmark{ }^{18}$ Clemens, N. T. and Narayanaswamy, V., "Low-Frequency Unsteadiness of Shock Wave/Turbulent Boundary Layer Interactions," Annual Review of Fluid Mechanics, Vol. 46, 2013, pp. 469-492.

${ }^{19} \mathrm{Wu}, \mathrm{M}$. and Martin, M. P., "Direct numerical simulation of supersonic turbulent boundary layer over a compression ramp," AIAA Journal, Vol. 45, No. 4, 2007, pp. 879-889. 